\title{
(6) OPEN ACCESS \\ Comparison of a powered bone marrow biopsy device with a manual system: results of a prospective randomised controlled trial
}

\author{
Christoph Marcus Bucher, ${ }^{1}$ Thomas Lehmann, ${ }_{1}^{1}$ André Tichelli, ${ }^{1}$ Alexander Tzankov, ${ }^{2}$ \\ Stephan Dirnhofer, ${ }^{1}$ Jakob Passweg, ${ }^{1}$ Alicia Rovó ${ }^{1}$
}

\begin{abstract}
- Additional supplementary files are published online only. To view these files please visit the journal online (http://dx. doi.org/10.1136/jclinpath2012-201167).

${ }^{1}$ Department of Hematology, University Hospital Basel, Basel, Switzerland

${ }^{2}$ Department of Pathology, University Hospital Basel, Basel, Switzerland
\end{abstract}

\section{Correspondence to} Dr Christoph Marcus Bucher, Department of Hematology, University Hospital Basel, Petersgraben 4, Basel 4031 Switzerland;

bucherc@uhbs.ch

$\overline{\mathrm{CB}}$ and $\mathrm{TL}$ contributed equally to this article

Received 14 September 2012 Revised 14 September 2012 Accepted 14 September 2012 Published Online First 26 October 2012

To cite: Bucher $\mathrm{CM}$ Lehmann T, Tichelli A, et al. J Clin Pathol 2013, 66, 2428.

\section{ABSTRACT}

The diagnostic and clinical usefulness of a powered bone marrow biopsy device (OnControl()) versus a standard manual device (TRAP Hospital System) was studied. Primary endpoints were biopsy quality and patient pain during the procedure. Fifty patients underwent a total of 60 procedures by three expert operators in a randomised stratified fashion. Baseline demographic and clinical parameters were similar in both groups. The usage of conscious sedation, dosage of lidocaine/pethidin was similar between groups. Biopsy quality was rated 'sufficient for diagnosis' in 24/30 in the control group and 25/30 in the powered group ( $p=0.74)$. Biopsy cylinder length, procedure time (from skin contact of the biopsy needle to placement of the biopsy cylinder in the formalin container) and patient reported pain during the procedure (T1), 15 min after the procedure (T2) and 3-5 days after the procedure (T3) there were comparable between groups. In the small subgroup of patients that did not receive conscious sedation ( $n=15$; manual 6 , powered 9) significantly lower median pain scores were observed with the powered system (median pain score 3 vs $7 ; p=0.015$ ). Patients were satisfied with either device whether sedation was used (sedation: median 9 for both groups, range 3-10 (manual) and 0-10 (powered)) no sedation (median 8 (manual) vs 9 (powered)). In summary bone marrow biopsies taken with the manual or powered device produce similar technical and clinical results. If no conscious sedation is used, pain during the procedure appears to be lower with the powered system. The use of a powered system seems to be justified in selected patients.

\section{INTRODUCTION}

Recent papers have indicated that a powered bone marrow biopsy device (On Control, Vidacare) is superior to manual devices to obtain marrow aspirates and bone marrow biopsies. ${ }^{1-4}$ An uncontrolled three-centre study ${ }^{1}$ using a first generation device reported fast insertion into the bone marrow cavity and short aspiration times paired with low insertional and aspirational pain scores in 55 patients. Follow up studies using a second generation device allows for the extraction of a biopsy core cylinder after aspiration. A randomised study by Berenson ${ }^{3}$ found that the new device produces significantly larger biopsies, shortens procedure time and reduces intermediate-term pain. Two reports by Swords ${ }^{2}{ }^{4}$ found significantly less insertional pain, significantly shortened procedure time, longer marrow biopsies and better operator satisfaction. However, crush artefacts were more abundant with the powered system.

Data from our department, ${ }^{5}$ a large retrospective study $^{6}$ and repeated surveys ${ }^{7}$ have indicated that the manual procedure is very safe and that the diagnostic yield is generally high. However, in a fraction of patients diagnostic yield is insufficient and could potentially be improved by larger biopsy specimens. The impact of the larger cylinders obtained with the powered device on their diagnostic value has not been addressed.

The primary goal of this study was to determine the clinical relevance of differences of quality of cylinders obtained either with a standard manual device (Hospital TRAPSYSTEM-HS Hospital Service S.p.A., Aprilia Italy $11 \mathrm{G} \times 100 \mathrm{~mm}$ ) or a powered device (OnControlTM, Vidacare, Shavano Park, Texas, USA $11 \mathrm{G} \times 103 \mathrm{~mm}$ ). Further, we wanted to address the hypothesis that the powered system is less painful during and after the intervention, potentially leading to improved patient satisfaction.

\section{METHODS}

\section{Trial design}

Prospective single centre non-blinded randomised trial.

\section{Patients}

The protocol was approved by the ethics committee of Basel and all patients signed informed consent (EKBB 88/11). Both hospitalised patients on the transplant ward and ambulatory patients in the clinic were eligible for inclusion. Recruitment started on May 5th 2011 and was concluded on the 8th of August 2011. Patients could be included and randomised more than once.

\section{End points}

Primary endpoint was diagnostic utility of the biopsy. This was judged by a pathologist unaware of the device used. If the pathologist judged that the material was sufficient to make a diagnosis the cylinder was rated 1 , in all other cases it was rated 0 . Secondary endpoints were cylinder length, number of marrow spaces, and presence of crush or aspiration artefacts. In addition, the quality of the aspirate was rated diagnostic or non-diagnostic. Clinical variables were patient pain during the procedure, $30 \mathrm{~min}$ after the procedure and 3-5 days after the procedure. Pain was rated on a visual analogue scale (VAS) from $0-10$ with 10 being maximal pain. The patient was also grading his 
overall satisfaction on a VAS with the procedure with 10 being maximally satisfied. Procedure time was measured from skin contact of the needle until the biopsy was ejected into the formalin (including aspiration time) by the assisting nurse using a chronometer. To compare the overall pain level during the current procedure with the pain level of previous procedures, patients were asked to indicate the overall pain and satisfaction with previous procedures.

\section{Inclusion/exclusion criteria}

Subjects with all of the following characteristics are eligible for study enrolment: age $>18$ years, $\geq$ one previous bone marrow procedure, INR $>1.4$, thrombocyte count $>10 \times 10^{9} / 1$, informed consent signed. Exclusion Criteria were: cognitive impairment, excessive tissue at anatomical landmarks, body mass index $(\mathrm{BMI})>35 \mathrm{~kg} / \mathrm{m}^{2}$, allergy to premedication, unable to lay flat in prone position.

\section{Sample size}

A preliminary study on 29 cases revealed that cylinders below $11 \mathrm{~mm}$ were most likely to be non-diagnostic (25\% vs $0 \%)$. Medium cylinder length at our centre was $12 \mathrm{~mm}$ (SD $6 \mathrm{~mm}$ ). Therefore, based on the previous reports and these data, we assumed that a median length of $18 \mathrm{~mm}$ with a SD of $9 \mathrm{~mm}$ should be achievable with the new device. With an $\alpha$ of 0.05 and a power level of 0.9 , a sample size of 28 patients per group was needed to reach the primary endpoint. Therefore, we chose to include 30 patients in each arm of the study.

\section{Operators}

Three experienced haematologists with $>200$ conventional biopsies performed. All operators were trained during three procedures with the powered device by training staff of Tinovamed.

\section{Device}

The powered device has been described previously. ${ }^{4}$ The manual device was a HS Trapsystem.

\section{Randomisation}

A Person unrelated to the procedures did the randomisation. For each operator, differently coloured, labelled and numbered envelopes containing sheets labelled with 'manual' or 'powered' were put into envelopes in a random fashion prior to initiation of the study. After informed consent was obtained the operator picked the next envelope with his assigned colour.

\section{Analgesia/conscious sedation}

Local analgesia was done with $2 \%$ subcutaneous lidocaine, conscious analgo-sedation was done according to the departmental standard with intravenous midazolam and intravenous pethidin. Dosages were at the discretion of the operator. Typically 10$20 \mathrm{ml}$ of $2 \%$ lidocaine, $20-50 \mathrm{mg}$ pethidin and $2-5 \mathrm{mg}$ midazolam were used. If patients did indicate that sedation was not wanted, only lidocaine was used.

\section{Statistical analysis}

Data were written into CRFs by the operators and entered into SPSS v21 by CMB. VAS scores and number of marrow spaces were considered as categorical data and analysed using $\chi^{2}$ and Mann-Whitney U test where appropriate. Drug dosages, cylinder length and procedure time were considered continuous data and analysed using students t-test. All $\mathrm{p}$ values are two-sided and were considered significant if $<0.05$.

\section{RESULTS}

\section{Patients}

Fifty out of fifty-eight screened subjects were randomised (see online supplementary figure S1). The 50 patients were randomised to receive a bone marrow aspirate and biopsy either with a manual device or the powered system. Forty-one patients received one procedure, eight patients received two procedures and one patient received three procedures. Demographic and clinical baseline data are shown in table 1 , figure 1 .

\section{Biopsy quality}

Biopsy quality was rated 'sufficient for diagnosis' in $24 / 30$ in the control group and $25 / 30$ in the powered Group $(p=0.74)$. Cylinder length was similar in both groups (figure $2 ; 14.2 \mathrm{~mm}$ $(5-37 \mathrm{~mm})$ vs $14.6 \mathrm{~mm}(6-27 \mathrm{~mm}), \mathrm{p}=0.79)$. Further parameters of biopsy quality studied were number of marrow spaces, crush artefacts and aspiration artefacts. The median number of marrow spaces was higher in the powered group than in the manual group ( $\mathrm{n}=8$ vs 6 ), but this difference was not statistically significant $(p=0.459)$. By contrast, crush artefacts and aspiration artifacts were slightly more frequent with the powered system than with the manual device ( $\mathrm{n}=17$ vs 12 and 14 vs 13 ), but again this did not reach statistical significance (crush artefacts $p=0.301$, aspiration artefacts $p=1.0$ ). Taken together these data suggest that the quality of biopsy cylinder is comparable using the manual and the powered device, thus our hypothesis that non-diagnostic biopsies could be reduced with the powered system was not confirmed. In both groups, the biopsy could be captured during the first attempt (table 2).

Aspirate quality was equal between groups. No difference in the number of sicca aspirates or in the quality of the smear was observed between groups (data not shown).

\section{Procedure time}

The procedure time was measured from skin contact of the biopsy needle to placement of the biopsy cylinder in the

Table 1 Baseline characteristics

\begin{tabular}{|c|c|c|c|}
\hline & Manual & Powered & \\
\hline $\mathrm{N}$ & 24 & 26 & \\
\hline Procedures & 30 & 30 & \\
\hline Age (median, range) & $56(20-69)$ & $58(18-66)$ & $p=0.98$ \\
\hline Body mass index $\left(\mathrm{kg} / \mathrm{m}^{2}\right)$ & $23(18-33)$ & $24(17-34)$ & $p=0.80$ \\
\hline Previous procedures (n) 1 & 2 & 2 & $p=0.41$ \\
\hline $2-6$ & 12 & 14 & \\
\hline$>6$ & 10 & 10 & \\
\hline Diagnosis ALL & 6 & 2 & $p=0.70$ \\
\hline AML & 5 & 9 & \\
\hline CML & 4 & 4 & \\
\hline MDS & 4 & 5 & \\
\hline MM & 3 & 3 & \\
\hline NHL & 6 & 4 & \\
\hline Other & 2 & 3 & \\
\hline No conscious sedation used & 6 & 9 & $p=0.552$ \\
\hline $\begin{array}{l}\text { Pain during previous procedure (median, } \\
\text { range) }\end{array}$ & $5(0-10)$ & $5(0-10)$ & $p=0.61$ \\
\hline $\begin{array}{l}\text { Previous experience with procedure } \\
\text { (median, range) }\end{array}$ & $5(0-10)$ & $5(0-10)$ & $p=0.28$ \\
\hline
\end{tabular}


Figure 1 Typical results using the manual and the powered device.



formalin container. Although intervention time tended to be shorter with the powered device, there was no significant difference between the groups in procedure time. With the manual system, median time was 180 s compared with 150 s with the powered system (range $80-480$ s vs $60-720$ s; $\mathrm{p}=0.947$ ).

\section{Patient pain}

To compare the pain that was experienced by the patients undergoing bone marrow biopsy, the patient was asked to

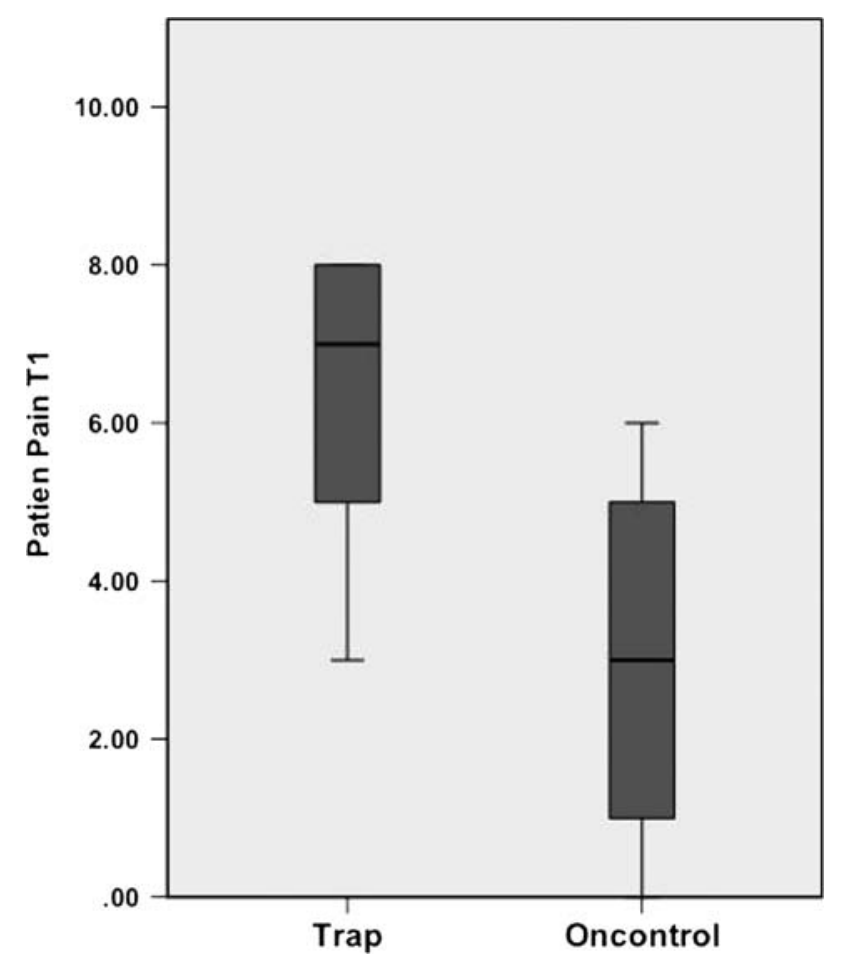

Figure 2 Patient pain in patients without conscious sedation. report his/her pain three times: Immediately after the procedure to give an overall score for the whole procedure (T1), $15 \mathrm{~min}$ after the procedure, if there was residual pain (T2) and 3-5 days after the procedure to report persisting pain (T3).

In all three time points there was no difference in patient reported median pain between the devices used (pain level manual vs powered T1: $2 / 10$ vs $1 / 10$, T2: $0 / 10$ vs $0 / 10$, T3: $0 /$ 10 vs $0 / 10 ; p=0.086, p=0.815, p=0.787$ respectively). Usage of conscious sedation (24/30 patients and $21 / 30$ patients respectively $(\mathrm{p}=0.56))$ and dosages of lidocaine $(10 \mathrm{ml}$ vs $13 \mathrm{ml}$ $\mathrm{p}=0.24)$, pethidin (23 $\mathrm{mg}$ vs $21 \mathrm{mg}, \mathrm{p}=0.68)$ and midazolam (2.5 mg vs $2.6 \mathrm{mg} \mathrm{p}=0.89$ ) was similar between groups.

At T2 10 Patients reported any pain. Five patients in the manual group had pain levels of $1(n=3), 2(n=1)$ and $3(n=1)$. The five patients with T2 pain in the powered device groups

Table 2 Results

\begin{tabular}{llll}
\hline & Manual & Powered & \\
\hline Cylinder diagnostic & $24 / 30$ & $25 / 30$ & $\mathrm{p}=0.74$ \\
$\begin{array}{l}\text { Cylinder length mm (median, } \\
\text { length) }\end{array}$ & 14.2 & 14.6 & $\mathrm{p}=0.79$ \\
Marrow spaces, median, (range) & $(5-37 \mathrm{~mm})$ & $(6-27 \mathrm{~mm})$ & \\
Fractured cylinder $\mathrm{n}$ & $6(1-15)$ & $8(1-20)$ & $\mathrm{p}=0.46$ \\
Aspiration artefacts n & $12(40 \%)$ & $17(57 \%)$ & $\mathrm{p}=0.301$ \\
Procedure time duration, in seconds & $13(43 \%)$ & $14(47 \%)$ & $\mathrm{p}=1.0$ \\
median, (range) & $180(80-480)$ & $150(60-720)$ & $\mathrm{p}=0.947$ \\
Patient pain (median; mean, range) & & & \\
$\quad$ & & & \\
$\quad$ T1 & $2: 3.2(0-10)$ & $1: 2.1(0-10)$ & $\mathrm{p}=0.09$ \\
$\quad$ T2 & $2: 0.28(0-3)$ & $1: 0.44(0-5)$ & $\mathrm{p}=0.82$ \\
$\quad$ T1 without conscious sedation & $0: 0.86(0-8)$ & $0: 1(0-8)$ & $\mathrm{p}=0.78$ \\
Patient satisfaction overall & $9(3-10)$ & $9(0-10)$ & $\mathrm{p}=0.69$ \\
Patient satisfaction without & $9(8-10)$ & $10(2-10)$ & $\mathrm{p}=0.12$ \\
sedation & & & \\
\hline
\end{tabular}


had scores of $1(n=1), 2(n=3)$ and $5(n=1) ; p=0.40$. At T3 the respective scores for manual and powered were $1(3 ; 1), 2$ $(0 ; 2), 3(1 ; 1), 5(1 ; 1), 8(1 ; 1) \mathrm{p}=0.70$. Four of these patients complained about relevant pain (two in each group), including two patients with post procedural haematoma. Interestingly, post procedural pain at T2 or T3 did not correlate with the use of conscious sedation. To control for individual pain perception patients were asked about pain levels in previous procedures. The median pain experienced during previous procedures was similar in both groups (manual median $=5$, powered $=6$; $\mathrm{p}=0.335$ ). Interestingly these values were significantly higher than values reported during the trial (manual median=2, powered median $=1$ ).

In the small subgroup of patients that did not receive sedation $(n=15$; manual 6 , powered 9) significantly lower median pain scores were observed with the powered system than with the manual device (median score 3 vs 7 respectively; $p=0.015$ ). No difference was observed in T2 and T3 between devices in this subgroup.

\section{Patient satisfaction}

Patients were very satisfied with either device whether sedation was used (median 9 for both groups, range 3-10 (manual) and 0-10 (powered)) or not (median 8 (manual) vs 9 (powered)). Patient satisfaction correlated loosely with $\mathrm{T} 1\left(\mathrm{R}^{2}\right.$ linear 0.059 and 0.459 for manual and powered, respectively) and procedure time $\left(\mathrm{R}^{2}\right.$ linear 0.508 and 0.271 ) without any of these correlations reaching statistical significance.

\section{Operator satisfaction}

Operator satisfaction was equally high with both devices (manual: median $8(6-10)$, powered $9(8-10) ; \mathrm{p}=0.213)$.

\section{Adverse events}

Adverse events were rare and could not be evaluated statistically. Two patients in the powered group reported painful haematoma at T3. One was clinically palpable and had a size of $2 \times 1 \mathrm{~cm}$. The other was minor. Both resolved. One additional patient in each group reported persisting pain at T3 (Level manual: 5, OnControl 8 ; but only while sitting down). One powered insertion needle broke during the attempt to extract the biopsy specimen.

\section{DISCUSSION}

This is the first independent prospective randomised trial of a powered biopsy system capable of aspiration and biopsy through the same puncture. Our results indicate that the manual system and the powered device produce comparable results. Our primary hypothesis, that the powered device would produce significantly larger biopsies and therefore help to avoid 'nondiagnostic' biopsies was not met. This is only seemingly in contrast with the reports published so far. Diagnostic usefulness was used as relevant parameter instead of biopsy volume, because if a minimal size requirement is met, additional marrow spaces provide no further diagnostic/staging benefit. In this context the usage of the powered device produced no diagnostic benefit in our setting. These results are not due to a training effect because all operators had at least three procedures done before starting the trial. In addition, no learning curve was observed during the trial in terms of procedure time or patient pain/satisfaction levels (data not shown). All operators are very experienced haematologists who did $>300$ of biopsies with the manual device before the trial started. Therefore, we can only speculate whether operators with less experience with the manual system would have produced different results. Patient ages and diagnoses as well as BMI values reported represent a typical mix that is seen in many academic teaching hospitals.

Previous studies have reported significantly shorter procedure times with the powered device. We were not able to reproduce this finding. This might be caused by our more clinically relevant definition of procedure time that included the time of aspiration. Although we did not control for aspiration time, it is unlikely that the results are caused by differences in aspiration time, as the study was stratified for operators and diagnoses were evenly distributed across groups. Patient reported pain levels and patient satisfaction were similar across groups. Given the unexpectedly high satisfaction of patients with the manual device, any new device would have to perform extremely well to be able to best this standard. However, patient pain levels were significantly lower in both groups than the levels patients reported from their memory. This indirectly confirms a finding from a previous study that found a significant positive correlation between patient information and pain. ${ }^{5}$ As per study protocol, patient information was extensive for the patients included in the trial. Additional reasons for lower pain could include standardised analgesia/sedation, more experienced operators and patients positive attitudes towards on protocol treatment. Qualitative differences of patient information have been reported to result in differences in pain perception for venipuncture. ${ }^{8}$ It is unlikely that in this study this effect was in play, as no standardised wording but routine language was used to inform patients.

Interestingly, the subgroup of patients who did not wish conscious sedation did report significantly lower pain levels when the powered device was used. Although numbers are small and this was not addressed directly by the study, this result could indicate that the use of a powered system could be justified in this subgroup of patients, potentially reducing duration of post biopsy bed rest and usage of resources.

Our trial has several important limitations. Although the design of the study is prospective randomised, the patients and the operators are not blinded. This could introduce a positive or negative effect for either device that could potentially be significant if blinding would be possible. Due to the design of the study patients attitudes towards either device can only be deducted from the overall satisfaction level. Because satisfaction level was very high in the baseline group, additional positive experiences in the experimental arm are hard if not impossible to quantify. For example patients with extremely hard bone were pleased by the obvious advantage through the powered device, but this is not reflected in the end points of the study. Overall, at about three times of the cost of a manual device, the powered device offered very limited advantages. Based on the data presented, outpatients who do not wish conscious sedation might be considered for the use of these devices.

\section{Take home messages}

- Manual and powered bone marrow biopsy devices produce similar result.

Acknowledgements We thank the study group team, all nurses of the transplant ward and ambulatory patients of the Haematology Department and the technicians of the Haematology Laboratory of the University Hospital of Basel for their collaboration in this study.

Contributors CMB designed the study, treated patients, analysed data and wrote the paper, TL treated patients and wrote the paper, AR initiated the project, treated 
patients and wrote the paper, ATzankov and SD analysed pathology samples, JP and ATichelli wrote the paper.

Competing interests None.

Patient consent Obtained.

Ethics approval Ethikkomission beider Basel, Basel, Switzerland.

Provenance and peer review Not commissioned; externally peer reviewed.

Open Access This is an Open Access article distributed in accordance with the Creative Commons Attribution Non Commercial (CC BY-NC 3.0) license, which permits others to distribute, remix, adapt, build upon this work non-commercially, and license their derivative works on different terms, provided the original work is properly cited and the use is non-commercial. See: http://creativecommons.org/licenses/by-nc/3.0/

\section{REFERENCES}

1 Cohen SC, Gore JM. Evaluation of a powered intraosseous device for bone marrow sampling. Anticancer Res 2008;28:3843-6.
2 Swords RT, Kelly KR, Cohen SC, et al. Rotary powered device for bone marrow aspiration and biopsy yields excellent specimens quickly and efficiently. I Clin Pathol 2010;63:562-5.

3 Berenson JR, Yellin O, Blumenstein B, et al. Using a powered bone marrow biopsy system results in shorter procedures, causes less residual pain to adult patients, and yields larger specimens. Diagn Pathol 2011;6:23.

4 Swords RT, Anguita J, Higgins RA, et al. A prospective randomised study of a rotary powered device (OnControl) for bone marrow aspiration and biopsy. I Clin Pathol 2011;64:809-13.

5 Degen C, Christen S, Rovo A, et al. Bone marrow examination: a prospective survey on factors associated with pain. Ann Hematol 2010;89:619-24.

6 Bain BJ. Bone marrow biopsy morbidity and mortality. Br I Haematol 2003;121:949-51.

7 Bain BJ. Morbidity associated with bone marrow aspiration and trephine biopsy-a review of UK data for 2004. Haematologica 2006;91:1293-4.

8 Ott J, Aust S, Nouri K, et al. An everyday phrase may harm your patients: the influence of negative words on pain during venous blood sampling. Clin I Pain 2012:28:324-8. 